\title{
Physical activity as a treatment for depressed adults
}

GE Mead

Professor of Stroke and Elderly Care Medicine, University of Edinburgh Edinburgh, UK

TITLE Facilitated physical activity as a treatment for depressed adults: randomised controlled trial

AUTHORS Chalder M,Wiles NJ, Campbell J et al.

JOURNAL BMJ 20I2; 344:e2758.

DECLARATION OF INTERESTS Professor Mead will receive a $£ 5,000$

Cochrane incentive grant to update their Review.

\author{
Correspondence to GE Mead \\ University of Edinburgh \\ Room SI642 \\ Royal Infirmary \\ Little France Crescent \\ Edinburgh EHI6 4SA \\ Scotland, UK
}

tel. $+44(0)$ I 3 I 242648 I

e-mail Gillian.E.Mead@ed.ac.uk
Physical inactivity and smoking are the two major risk factors for non-communicable diseases worldwide,' and so increasing physical activity for the population is important for public health.

There is an extensive literature, going back many years, that suggests that exercise improves depression through a number of different mechanisms. The most recent Cochrane Review reported that exercise improved mood in people with depression, but the effect size was only moderate, and when lower quality trials were excluded, the effect size was only small. ${ }^{2}$ The Review authors recommended that further trials of exercise for depression were needed, and that these needed to be methodologically robust.

\section{SUMMARY}

Chalder et al. $^{3}$ recently published a landmark trial in which they recruited $36 \mathrm{I}$ adults aged 18 to 69 years of age, with a clinical diagnosis of depression, from general practices in Bristol and Exeter. Participants were randomised to usual care plus an intervention designed to increase physical activity, or usual care alone. The intervention consisted of three face-to-face sessions and ten telephone calls with a trained physical activity facilitator over eight months. The intervention was based on theory and aimed to provide individually tailored support and encouragement to engage in physical activity. The primary outcome was the Beck depression inventory at four months after randomisation. The trialists reported that adherence to the intervention was good, and that at the end of the trial, levels of selfreported physical activity were higher in the intervention group. Despite good adherence and an increase in selfreported activity, however, there was no difference in depression scores between the two groups, and no difference in antidepressants usage (one of the secondary outcomes) at the end of the trial.

\section{OPINION}

How should the results be interpreted, given that the Cochrane Review suggests that exercise might have a small effect on mood in people with depression? This trial was methodologically robust, and was substantially larger than previous trials. Furthermore, participants could enter the trial only if they had an International Classification (ICD) 10 diagnosis of depression, whereas in some studies included in the Cochrane Review, ${ }^{2}$ the diagnosis of depression was made using a cut-off point on a mood scale, rather than by clinical diagnostic interview. Another explanation is that the quality of 'usual care' was already high and so the addition of exercise did not make much difference. Finally, it is still possible that physical activity might improve depression, but it may need to be at a higher 'dose' than was achieved in the trial.

Based on the results of this clinical trial, clinicians should not advise people with depression that physical activity will increase their chances of recovering from depression. Nevertheless, clinicians and policy makers should ensure that they promote the public health message that physical activity has multiple health benefits. ${ }^{3}$

\section{REFERENCES}

I Lee IM, Shiroma EG, Lobela F et al. Effect of physical inactivity on major non-communicable diseases worldwide: an analysis of burden of disease and life expectancy. Lancet 2012; 380:219-29. http://dx.doi.org/10.1016/S0140-6736(12)61031-9

2 Rimer J, Dwan K, Lawlor DA et al. Exercise for depression. Cochrane Database Syst Rev 2012; 7:CD004366. http://dx.doi. org/I0.1002/I465I858.CD004366.pub5

3 Chalder M,Wiles NJ, Campbell J et al. Facilitated physical activity as a treatment for depressed adults: randomised controlled trial. BMJ 2012; 344:e2758. http://dx.doi.org/I0.II36/bmj.e2758 